\title{
EQUIPAMENTOS DAS ACADEMIAS DA TERCEIRA IDADE: INFLUÊNCIA SOBRE O COMPORTAMENTO DA PRESSÃO ARTERIAL E DA FREQUÊNCIA CARDÍACA DE IDOSOS
}

\author{
Equipment in senior gyms: influence on the \\ behavior of blood pressure and heart rate of elderlies
}

Sillas Oliveira Leonel Júniora, Mateus Dias Antunesa ${ }^{a}$ Ramon Gustavo de Moraes Ovandoa, Ana Paula
Serra de Araújo ${ }^{a}$, Flávio Bortolozzia, Marcelo Picinin Bernucia, Sônia Maria Marques Gomes Bertolini

OBJETIVO: Analisar o comportamento da pressão arterial e da frequência cardíaca de idosos praticantes de exercícios físicos em academias da terceira idade de Maringá, Paraná. MÉTODO: Foram avaliados 70 idosos voluntários, sendo 41 mulheres e 29 homens, com idade média de 67,2 \pm 6,6 e 65,9 \pm 12,3 anos, respectivamente. Realizaram três minutos de exercício nos equipamentos selecionados, avaliados por meio de um frequencímetro e pelo método auscultatório, com auxílio de um esfigmomanômetro. RESULTADOS: Os valores elevados de frequência cardíaca foram registrados no multiexercitador. Quer seja na frequência cardíaca, quer seja na pressão arterial, houve diferenças significantes nas médias dos valores encontrados na avaliação inicial, comparadas às médias da avaliação final $(p<0,05)$. CONCLUSÕES: A pressão arterial eleva-se dentro dos limites fisiológicos estabelecidos para os idosos, podendo tais atividades serem consideradas seguras para essa população.

PALAVRAS-CHAVE: frequência cardíaca; atividade motora; envelhecimento; promoção da saúde.

OBJECTIVE: To analyze the behavior of blood pressure and heart rate in elderly people practicing physical exercises in Maringá, Paraná, Brazil. METHODS: 70 elderly volunteers were evaluated, 41 women and 29 men, with a mean age of $67.2 \pm 6.6$ and 65.9 \pm 12.3 years, respectively. They performed three minutes of exercise on the selected equipment, evaluated by means of a frequency meter and by the auscultatory method, with the aid of a sphygmomanometer. RESULTS: High heart rate values were recorded in the multi-exerciser. In both the heart rate and the blood pressure, there were significant differences in the means of the values found in the initial evaluation, compared to the means of the final evaluation $(p<0.05)$. CONCLUSIONS: Blood pressure rises within the physiological limits established for the elderly, and these activities may be considered safe for this population.

KEYWORDS: heart rate; motor activity; aging; health promotion.

aCentro Universitário de Maringá - Maringá (PR), Brasil.

Dados para correspondência

Mateus Dias Antunes - Centro Universitário de Maringá - Avenida Guedner, 1610 - Jardim Aclimação - CEP: 87050-900 - Maringá (PR), Brasil E-mail: mateus_antunes03@hotmail.com

Recebido em: 08/06/2017. Aceito em: 14/09/2017

DOI: 10.5327/Z2447-211520171700042 


\section{INTRODUÇÃO}

Com o aumento do número de idosos e da expectativa de vida no país, crescem também a prevalência de doenças crônicas não transmissíveis (DCNT) e a preocupação com a elaboração e com a implementação de estratégias de promoção da saúde voltadas para atender a necessidade dessa população. Atualmente, uma das estratégias de promoção da saúde mais recomendadas para a população idosa é o incentivo às práticas corporais e atividades físicas. $\mathrm{O}$ incentivo à prática de atividade física regular e a elaboração e execução de programas de atividade para idosos têm sido vistos como algumas das principais ações do setor de saúde para promoção da prevenção de DCNT (como hipertensão arterial sistêmica, diabetes, osteoporose e obesidade) entre idosos, e para proporcionar a eles uma vida mais ativa e independente, com maior autonomia e melhor qualidade de vida. ${ }^{1,2}$

Entre as estratégias e programas existentes incentivados pelo Ministério da Saúde do Brasil que visam a estimular a prática de atividades físicas pela população idosa, tem-se a implantação de academias da terceira idade (ATI), também denominadas academias ao ar livre (AAL) ou academias da saúde. Essas academias são instaladas em espaços públicos urbanos como praças e bosques e equipadas com aparelhos de ginástica que não utilizam cargas para a prática de exercício físico, apenas a força do próprio corpo para exercícios de musculação, mobilidade articular e alongamento. ${ }^{3}$

De modo geral, as ATI constituem um sistema para a prática de atividade física que se adapta ao usuário, à sua capacidade física e ao seu ritmo, criando resistência e gerando benefício para os sistemas corpóreos personalizados, como o cardiovascular e o musculoesquelético. ${ }^{3}$ Além disso, essas academias incentivam a prática de atividades físicas regulares pelo seu fácil acesso, estimulam a inclusão social, melhoram a autoestima, a qualidade de vida e a saúde daqueles que as frequentam. ${ }^{4}$

As ATI são indicadas para uso de indivíduos com idade acima de 12 anos, principalmente idosos, ${ }^{3}$ que são o grupo populacional mais beneficiado pela prática de atividades físicas regulares, devido às alterações morfofisiológicas advindas do envelhecimento do organismo humano e que podem ser minimizadas pelos exercícios. ${ }^{4}$

O conhecimento e a monitorização de parâmetros cardiovasculares de idosos durante a utilização dos equipamentos disponíveis nas ATI, em especial aqueles que geram resistência física, tornam-se de grande relevância. Poderão contribuir não só para a análise de como o uso desses equipamentos influencia parâmetros cardiovasculares, mas também para que se prescreva com segurança a prática de exercícios físicos nessas academias para idosos. Dessa forma, o presente estudo objetivou analisar o comportamento das variáveis cardiovasculares, como pressão arterial (PA) e frequência cardíaca (FC), de idosos praticantes de exercícios físicos em ATI do município de Maringá, noroeste do Paraná.

\section{MÉTODOS}

O presente estudo, realizado após aprovação do Comitê de Ética e Pesquisa do Centro Universitário de Maringá (UNICESUMAR), Parecer no 408.476/2013, caracterizouse como uma pesquisa intervencional de natureza quantitativa. Foi realizado em 8 das 57 ATI de Maringá, sendo elas: Parigot de Souza, Quebec, Parque do Ingá, Parque Alfredo Nyeffeller, Mandacaru, Vila Olímpica, Centro Esportivo do Jardim Alvorada e Praça das Américas. Foram escolhidas as ATI que, quando visitadas anteriormente, mostraram-se as mais frequentadas pela população idosa.

A população foi constituída por indivíduos com idade igual ou superior a 55 anos, de ambos os sexos, praticantes de exercícios físicos nas ATI de Maringá há pelo menos 3 meses por no mínimo 2 vezes por semana. Para determinação do tempo de exercício realizado em cada equipamento, foi realizada observação in loco da rotina dos idosos nas ATI. A coleta de dados foi realizada no período compreendido entre outubro e novembro de 2014, totalizando 30 dias, e foi feita por uma equipe interdisciplinar composta por dois fisioterapeutas, uma bióloga e uma nutricionista. Para se alcançar os objetivos propostos, cada profissional ficou responsável pela coleta de dados em duas ATI. Os pesquisadores realizaram plantões de segunda a domingo nas ATI no período matutino (das $6 \mathrm{~h} 30$ às $9 \mathrm{~h}$ ) e no período vespertino (das $15 \mathrm{~h}$ às $18 \mathrm{~h}$ ). Durante os plantões, os profissionais abordavam individualmente os idosos que chegavam e os convidavam a participar do estudo, explicando os objetivos da pesquisa, sua finalidade e como a mesma ocorreria. Também foi solicitada a assinatura do termo de consentimento livre e esclarecido (TCLE).

Ao concordarem em participar do estudo, os idosos foram submetidos inicialmente a uma entrevista estruturada, constando de questões sobre: idade (em anos completos), sexo, uso da ATI (em meses) e frequência dos equipamentos utilizados na ATI (vezes por semana). Posteriormente, foram instruídos a realizar atividades físicas em seis dos dez equipamentos das ATI, de forma aleatória, para não influenciar nos resultados da pesquisa, sendo os equipamentos selecionados aqueles que geram resistência musculoesquelética, como o simulador de caminhada, o esqui, a remada, o surf, a cavalgada e o multiexercitador. Os voluntários foram instruídos a realizar três minutos ininterruptos de atividade em cada um dos seis equipamentos. O tempo entre os exercícios em cada equipamento dependeu do retorno da $\mathrm{FC}$ aos valores iniciais, 
o que variou de três a quatro minutos de repouso, após execução da atividade em cada equipamento. Os idosos foram ainda instruídos a seguir seu ritmo costumeiro de execução das atividades nos equipamentos pesquisados.

Antes de iniciar a atividade em cada um dos equipamentos, os pesquisadores aferiram a PA e a FC de cada voluntário. Após três minutos de exercício, os voluntários foram instruídos a interromper a prática e tiveram os parâmetros supracitados novamente mensurados, sendo então solicitados a iniciar atividade no equipamento subsequente. A resposta aguda aos exercícios realizados em cada aparelho foi avaliada por meio de um cardiofrequencímetro Polar $^{\circledR}$ e os valores da pressão arterial sistólica (PAS) e diastólica (PAD) foram obtidos pelo método auscultatório com o auxílio de um esfigmomanômetro, seguindo os parâmetros estabelecidos pelas V Diretrizes Brasileiras de Hipertensão Arterial. ${ }^{5}$

Para controle do tempo, foi utilizado um cronômetro digital; já para determinação do tempo de execução da atividade física em cada aparelho das ATI e tempo de repouso após execução, foi realizado um estudo piloto que contou com a participação voluntária de dez indivíduos idosos praticantes de atividades físicas em ATI de Maringá.

Para o melhor conhecimento da intensidade dos exercícios realizados nos diferentes equipamentos, foi estimada, segundo a equação de Tanaka et al., ${ }^{6}$ a frequência cardíaca teórica [\%FCmáx. $=208-0,7 \times$ idade] e o respectivo valor percentual para cada um dos sujeitos avaliados [\%FCmáx. = FCmédia/FCmáx.T] durante a sessão de exercício.

O critério de classificação utilizado no presente estudo foi proposto pelo The American College of Sport Medicine (ACSM) ${ }^{7,8}$ Essa classificação é baseada em seis categorias, conforme Tabela 1.

Os dados coletados foram inseridos em planilhas e apresentados por meio de estatísticas descritivas, como tabelas, média e desvio padrão. Com o teste de Kolmogorov-Smirnov, foi observada a não normalidade dos dados e em seguida foi aplicado o teste de Wilcoxon para verificar as diferenças entre as variáveis. O nível de significância adotado foi de $5 \%(\mathrm{p}<0,05)$.

\section{RESULTADOS}

Dos 70 indivíduos avaliados, $58,6 \%(\mathrm{n}=41)$ eram do sexo feminino e $41,4 \%(n=29)$ eram do sexo masculino. A média de idade dos idosos foi de $67,5 \pm 6,3$ anos, sendo que a média de idade dos homens foi de $65,9 \pm 12,3$ anos e, das mulheres, de 67,2 \pm 6,6 anos (Tabela 1).

Ao avaliar a FC dos idosos de ambos os sexos em diferentes equipamentos das ATI, ficou evidenciada diferença significativa entre os momentos inicial e final das avaliações em todos os equipamentos (Tabela 2).
A Tabela 3 mostra a distribuição dos equipamentos segundo os parâmetros de intensidade do esforço, baseados na percentagem da FC máxima. Foi possível notar que os exercícios realizados na maioria dos equipamentos, por ambos os sexos, foram de intensidade moderada.

Ao avaliar a PAS e PAD nos idosos, tanto homens quanto mulheres, em diferentes equipamentos das ATI, ficou evidenciada diferença significativa entre as avaliações em todos os equipamentos (Tabelas 4 e 5).

\section{DISCUSSÃO}

Não foram encontrados estudos nacionais e internacionais que verificam os parâmetros cardiovasculares durante a prática de atividade física nas ATI. No presente estudo, em relação à $\mathrm{FC}$ inicial em cada equipamento, observou-se em ambos os sexos uma pequena variação entre os equipamentos, o que pode ser justificado pela menor capacidade de adaptação e recuperação ao exercício inerente ao processo de envelhecimento. ${ }^{9}$ Ao se considerar o sexo, verifica-se na Tabela 2 que a FC das mulheres, no momento inicial de cada exercício, é maior, o que está de acordo com a literatura sobre a fisiologia cardiovascular. ${ }^{10}$ Esse fato é justificado por níveis tipicamente mais altos de aptidão aeróbia no sexo masculino do que no feminino.

As alterações da FC e da PA que ocorrem durante o exercício refletem o tipo e a intensidade do exercício realizado, sua duração e as condições ambientais sob as quais o trabalho foi realizado. ${ }^{11}$ Foi possível observar que os valores mais elevados de FC foram registrados no multiexercitador, na cavalgada e no surfe (Tabela 2).

$\mathrm{Na}$ Tabela 3, os resultados mostram que a intensidade do esforço, avaliado por meio da variável hemodinâmica FC na maioria dos equipamentos da ATI ( $83 \%$ dos casos) foi fisiologicamente segura e suficientemente moderada para induzir possíveis adaptações no sistema cardiovascular. Apesar do

Tabela 1 Classificação da frequência cardíaca proposta pela The American College of Sport Medicine.

\begin{tabular}{l|c} 
Intensidade & $\%$ da frequência cardíaca máxima \\
\hline Muito leve & Abaixo de 35 \\
\hline Leve & Entre 35 e 54 \\
\hline Moderada & Entre 55 e 69 \\
\hline Alta & Entre 70 e 89 \\
\hline Muito alta & Igual ou acima de 90 \\
\hline Máxima & 100
\end{tabular}


multiexercitador promover, em ambos os sexos, as maiores elevações da FC, os valores ainda são considerados um esforço de moderada intensidade. Na literatura, existe relato de que exercícios em intensidade moderada (40 a $60 \%$ do consumo máximo de oxigênio - VO2 máx.) são tão efetivos quanto exercícios de intensidade vigorosa (mais de $60 \%$ do VO2 máx.) em relação ao efeito hipotensor a longo prazo. ${ }^{12}$

No que se refere à resposta hemodinâmica aguda do exercício físico, um dos fatores que influenciam no aumento da PA é o aumento da FC. ${ }^{13}$ Conforme pode ser observado na

Tabela 2 Distribuição da frequência cardíaca na avaliação inicial e final dos idosos praticantes de atividades físicas nas academias da terceira idade.

\begin{tabular}{|c|c|c|c|}
\hline \multirow{3}{*}{ Equipamentos } & \multicolumn{3}{|c|}{ Frequência cardíaca } \\
\hline & \multicolumn{2}{|c|}{ AVI $(n=41)$ AVF $(n=41)$} & \multirow{2}{*}{ Valor $\mathrm{p}$} \\
\hline & Média \pm DP & Média \pm DP & \\
\hline \multicolumn{4}{|l|}{ Feminino } \\
\hline Simulador de caminhada & $72,1 \pm 10,0$ & $82,8 \pm 10,9$ & $<0,001^{*}$ \\
\hline Esqui & $73,6 \pm 10,0$ & $88,9 \pm 15,3$ & $<0,001^{*}$ \\
\hline Remada & $76,5 \pm 9,7$ & $93,6 \pm 16,9$ & $<0,001^{*}$ \\
\hline Surfe & $77,5 \pm 10,4$ & $95,2 \pm 18,5$ & $<0,001^{*}$ \\
\hline Cavalgada & $77,9 \pm 11,3$ & $93,5 \pm 16,0$ & $<0,001^{*}$ \\
\hline Multiexercitador & $78,2 \pm 11,8$ & $99,0 \pm 20,1$ & $<0,001^{*}$ \\
\hline \multicolumn{4}{|l|}{ Masculino } \\
\hline Simulador de caminhada & $70,0 \pm 9,0$ & $80,6 \pm 11,3$ & $<0,001^{*}$ \\
\hline Esqui & $71,5 \pm 11,8$ & $86,0 \pm 17,1$ & $<0,001^{*}$ \\
\hline Remada & $72,1 \pm 10,9$ & $92,9 \pm 18,1$ & $<0,001^{*}$ \\
\hline Surf & $73,8 \pm 10,3$ & $92,0 \pm 20,1$ & $<0,001^{*}$ \\
\hline Cavalgada & $73,3 \pm 12,5$ & $91,5 \pm 13,3$ & $<0,001^{*}$ \\
\hline Multiexercitador & $75,7 \pm 12,7$ & $94,5 \pm 20,1$ & $<0,001^{*}$ \\
\hline
\end{tabular}

AVI: avaliação inicial; AVF: avaliação final; DP: desvio padrão; *Valor p significativo pelo teste Wilcoxon pareado considerando nível de significância de 5\%.

Tabela 3 Intensidade de esforço dos idosos em relação à frequência cardíaca nos equipamentos das academias da terceira idade.

\begin{tabular}{l|c|c|c|c}
\multirow{2}{*}{ Equipamentos } & \multicolumn{2}{|c}{ Feminino } & \multicolumn{2}{c}{ Masculino } \\
\cline { 2 - 5 } & $\begin{array}{c}\text { \% da frequência } \\
\text { cardíaca máxima }\end{array}$ & $\begin{array}{c}\text { Intensidade } \\
\text { de esforço }\end{array}$ & $\begin{array}{c}\text { \% da frequência } \\
\text { cardíaca máxima }\end{array}$ & $\begin{array}{c}\text { Intensidade } \\
\text { de esforço }\end{array}$ \\
\hline Simulador de caminhada & 51,50 & Leve & 50,13 & Leve \\
\hline Esqui & 55,30 & Moderada & 55,10 & Moderada \\
\hline Cavalgada & 58,16 & Moderada & 56,60 & Moderada \\
\hline Remada & 58,22 & Moderada & 57,23 & Moderada \\
\hline Surf & 59,22 & Moderada & 58,78 & Moderada \\
\hline Multiexercitador & 61,58 & Moderada & &
\end{tabular}

Nota: Segundo critérios da The American College of Sport Medicine. 
Tabela 4 Distribuição da pressão arterial sistólica na avaliação inicial e final dos idosos praticantes de atividades físicas nas academias da terceira idade.

\begin{tabular}{|c|c|c|c|}
\hline \multirow{3}{*}{ Equipamentos } & \multicolumn{3}{|c|}{ Pressão arterial } \\
\hline & \multicolumn{2}{|c|}{ AVI $(n=41)$ AVF $(n=41)$} & \multirow{2}{*}{ Valor $\mathrm{p}$} \\
\hline & Média \pm DP & Média \pm DP & \\
\hline \multicolumn{4}{|l|}{ Feminino } \\
\hline Simulador de caminhada & $120,5 \pm 11,6$ & $128,3 \pm 12,6$ & $<0,001^{*}$ \\
\hline Esqui & $122,0 \pm 12,9$ & $123,7 \pm 20,8$ & $<0,001^{*}$ \\
\hline Remada & $119,0 \pm 10,4$ & $126,3 \pm 11,6$ & $<0,001^{*}$ \\
\hline Surfe & $123,4 \pm 10,2$ & $127,6 \pm 13,4$ & $<0,001^{*}$ \\
\hline Cavalgada & $123,4 \pm 8,5$ & $127,1 \pm 10,5$ & $<0,001^{*}$ \\
\hline Multiexercitador & $124,6 \pm 10,5$ & $129,5 \pm 10,7$ & $<0,001^{*}$ \\
\hline \multicolumn{4}{|l|}{ Masculino } \\
\hline Simulador de caminhada & $129,3 \pm 20,0$ & $134,8 \pm 20,3$ & $<0,001^{*}$ \\
\hline Esqui & $130,3 \pm 20,4$ & $135,2 \pm 18,6$ & $<0,001^{*}$ \\
\hline Remada & $128,6 \pm 20,0$ & $134,1 \pm 21,6$ & $<0,001^{*}$ \\
\hline Surf & $129,7 \pm 21,1$ & $134,8 \pm 19,0$ & $<0,001^{*}$ \\
\hline Cavalgada & $129,3 \pm 18,3$ & $136,1 \pm 20,1$ & $<0,001^{*}$ \\
\hline Multiexercitador & $131,7 \pm 18,1$ & $137,6 \pm 19,6$ & $<0,001^{*}$ \\
\hline
\end{tabular}

AVI: avaliação inicial; AVF: avaliação final; DP: desvio padrão; *Valor p significativo pelo teste Wilcoxon pareado considerando nível de significância de 5\%.

Tabela 5 Distribuição da pressão diastólica na avaliação inicial e final dos idosos praticantes de atividades físicas nas academias da terceira idade.

\begin{tabular}{|c|c|c|c|}
\hline \multirow{3}{*}{ Equipamentos } & \multicolumn{3}{|c|}{ Pressão arterial } \\
\hline & \multicolumn{2}{|c|}{ AVI $(n=41)$ AVF $(n=41)$} & \multirow{2}{*}{ Valor $p$} \\
\hline & Média \pm DP & Média \pm DP & \\
\hline \multicolumn{4}{|l|}{ Feminino } \\
\hline Simulador de caminhada & $74,9 \pm 9,3$ & $80,0 \pm 10,7$ & $0,003^{*}$ \\
\hline Esqui & $78,5 \pm 7,6$ & $82,7 \pm 8,7$ & $0,031^{*}$ \\
\hline Remada & $75,6 \pm 9,2$ & $80,0 \pm 8,9$ & $0,022^{*}$ \\
\hline Surfe & $76,0 \pm 8,1$ & $81,0 \pm 8,6$ & $0,037^{*}$ \\
\hline Cavalgada & $77,3 \pm 9,5$ & $82,2 \pm 7,2$ & $0,042^{*}$ \\
\hline Multiexercitador & $76,8 \pm 8,5$ & $83,7 \pm 8,0$ & $0,000^{*}$ \\
\hline \multicolumn{4}{|l|}{ Masculino } \\
\hline Simulador de caminhada & $79,7 \pm 12,1$ & $84,5 \pm 13,3$ & $0,015^{*}$ \\
\hline Esqui & $78,3 \pm 11,7$ & $82,8 \pm 13,3$ & $0,013^{*}$ \\
\hline Remada & $78,6 \pm 11,3$ & $83,1 \pm 12,0$ & $0,024^{*}$ \\
\hline Surf & $79,8 \pm 13,9$ & $83,1 \pm 11,7$ & $0,044^{*}$ \\
\hline Cavalgada & $80,0 \pm 12,9$ & $85,5 \pm 9,9$ & $0,048^{*}$ \\
\hline Multiexercitador & $81,4 \pm 12,5$ & $87,6 \pm 11,2$ & $0,002^{*}$ \\
\hline
\end{tabular}

AVI: avaliação inicial; AVF: avaliação final; DP: desvio padrão; *Valor p significativo pelo teste Wilcoxon pareado considerando nível de significância de 5\%. 
Tabela 3, os valores de PAS aumentaram relativamente em todos os equipamentos, para ambos os sexos, atingindo seu valor máximo no multiexercitador. Notou-se, ainda, que foram registrados, em ambos os sexos e em todos os equipamentos, diferenças estatisticamente significativas entre os valores médios da PAS na avaliação inicial e os valores da PAS na avaliação final. Em nenhum equipamento os valores da PA foram considerados de risco, sendo o valor máximo encontrado para a PAS 137,6 mmHg e, para a PAD, 87,6 mmHg.

Em relação ao comportamento dos parâmetros cardiovasculares dos idosos praticantes de atividades físicas em ATI, ainda existem poucas evidências. Esteves et al., ${ }^{4}$ após um programa sistematizado de atividades físicas durante seis meses na ATI, verificaram importantes reduções nos níveis pressóricos, o que foi relatado inclusive pelos próprios usuários. As modalidades de exercícios realizados nas ATI no momento da pesquisa considerando o tipo de contrações realizadas (predominantemente dinâmicas), a moderada carga externa (peso do próprio corpo e dos equipamentos) e o número reduzido de grupos musculares solicitados simultaneamente ${ }^{13}$ - podem explicar a menor resposta fisiológica ao esforço.

Os resultados da FC máxima e, consequentemente, da percentagem da FC máxima, apesar de estimados, permitiram observar que em ambos os sexos o simulador de caminhada não permitiu que os sujeitos alcançassem a intensidade requerida para a indução de alterações fisiológicas significativas; ${ }^{14}$ isso é, os idosos não atingiram 55\% da FC máxima. No entanto,é importante ressaltar que esses equipamentos visam não necessariamente ao desenvolvimento da capacidade aeróbia, mas sim melhores escores em variáveis motoras como agilidade e equilibrio. ${ }^{14}$ Os exercícios realizados no equipamento multiexercitador foram aqueles que induziram maiores elevações da FC e da PA, estando provavelmente relacionados com a maior massa muscular ativa. Nesse sentido, vale destacar que a quantidade e a qualidade de exercício necessárias para adultos idosos parecem associar-se a um menor risco de doenças crônicas degenerativas e à melhoria da aptidão metabólica.

Embora o tempo de exercício realizado em cada equipamento tenha sido estabelecido após observação in loco da rotina dos idosos nas ATI, destaca-se esse aspecto como um fator que pode implicar a generalização dos resultados, uma vez que nesses locais não existe, na maior parte do tempo, profissionais que possam orientar a realização desses exercícios de forma sistemática, com controle do tempo, frequência e intensidade. Isso é, pode ser que existam idosos realizando exercícios em um único equipamento durante um ou até mesmo dez minutos. Outro ponto que deve ser enfatizado é que a amostra foi composta por idosos praticantes de atividades físicas nas ATI há no mínimo três meses. Esse fato pode ser apontado como limitação do estudo, que não contou com a existência de um grupo controle constituído por idosos insuficientementes ativos.

\section{CONCLUSÃO}

$\mathrm{O}$ comportamento da $\mathrm{FC}$ indicou que a intensidade de esforço durante os exercícios praticados nos equipamentos das ATI é predominantemente moderada. Além disso, tanto a PAS como a PAD elevam-se dentro dos limites fisiológicos estabelecidos para os idosos, podendo tais atividades serem consideradas seguras para essa população.

Os achados da presente pesquisa sugerem que, embora os exercícios praticados nas ATI não visem necessariamente ao desenvolvimento da capacidade aeróbia, seria melhor se fossem incluídos equipamentos com características semelhantes ao multiexercitador, para que exercícios de intensidades moderadas sejam praticados por uma maior quantidade de idosos que frequentam as ATI.Todavia, novos estudos de intervenção controlados devem ser realizados para verificação dos efeitos crônicos, na FC e na PA, dos exercícios praticados em ATI pelos indivíduos acima de 60 anos.

\section{AGRADECIMENTOS}

Ao Instituto Cesumar de Ciência,Tecnologia e Inovação (ICETI) e à Coordenação de Aperfeiçoamento de Pessoal de Nível Superior (CAPES) pela concessão de bolsas de auxílio à pesquisa.

\section{CONFLITO DE INTERESSES}

Os autores declaram não haver conflito de interesses.

\section{REFERÊNCIAS}

1. Salvador EP, Florindo AA, Reis RS, Costa EF. Percepção do ambiente e prática de atividade física no lazer entre idosos. Rev Saúde Pública. 2009;43(6):972-980

2. Silva MC, Silva AB, Amorim TEC. Condições de espaços públicos destinados a prática de Atividade Físicas na cidade de Pelotas/RS/ Brasil. Rev Bras Ativ Fis e Saúde. 2012;17(1):28-32.
3. Lima FLR. Percepção do esforço em idosos nas academias ao ar livre. Rev Bras Prescr Fisiol Exerc. 2013;7(37):55-64

4. Esteves JVDC, Andreato LV, Pastório JJ, Versuti JKB, Almeida HC, Moraes SMF. $O$ uso de academias da terceira idade por idosos modifica parâmetros morfofuncionais? Acta Sci Health Sci. 2012;34(1):31-8.

5. Sociedade Brasileira de Cardiologia. V Diretrizes Brasileiras de Hipertensão Arterial. Arq Bras Cardiol. 2007;89(3):1-55. 
6. Tanaka H, Monahan KD, Seals DR. Age-predicted maximal heart rate revisited. J Am Coll Cardiol. 2001;37(1):153-6.

7. American College Sport Medicine. Position stand on the recommended cardiorespiratory and muscular fitness, and flexibility in healthy adults. Med Sci Sports Exerc. 1998;30(6):975-91.

8. American College Sports Medicine. ACSM's health-related physical fitness assessment manual. Philadelphia: Lippincott Williams \& Wilkins; 2004.

9. Matsudo SM, Matsudo VKR. Prescrição de exercícios e benefícios da atividade física na terceira idade. Rev Bras Ciên Mov. 1992;6(4):19-30.

10. Powers SK, Howley ET. Fisiologia do Exercício. São Paulo: Manole; 2009.
11. Sydó N, Abdelmoneim SS, Mulvagh SL, Merkely B, Gulati M, Allison TG Relationship between exercise heart rate and age in men vs women. Mayo Clinic Proceedings. 2014;89:1664-72.

12. Pescatello L. Exercise and Hypertension: Recent Advances in Exercise Prescription. Curr Hypertens. 2005;7(4):281-6.

13. Overend TJ, Versteegh TH, Thompson E, Birmingham TB, Vandervoort AA. Cardiovascular stress associated with concentric and eccentric isokinetic exercise in young and older adults. J Gerontol. 2000;55:B177-82.

14. Bertolini SMMG, Manueira P. Equilíbrio estático e dinâmico de idosos praticantes de atividades físicas em Academias da Terceira Idade. ConScientiae Saúde. 2013;12(3):432-8. 